\title{
Association of Neck, Wrist and Hip Circumferences with Kidney Function in Children and Adolescents: The CASPIAN- V Study
}

\author{
(1) Mehryar Mehrkash1', (1) Ramin Heshmat², (1) Mostafa Qorbani33, (1) Mohammad Esmaeil Motlagh4, \\ (1) Shirin Djalalinia5, (1) Sara Zamani6, (1) Majzoubeh Taheri1', (1) Gita Shafiee², (1) Armita Mahdavi-Gorabi², \\ (1) Azadeh Aminianfar77, (1) Tahereh Aminaei¹, (1) Roya Kelishadi \\ 1/sfahan University of Medical Sciences, Child Growth and Development Research Center, Research Institute for Primordial Prevention of \\ Non-communicable Disease, Department of Pediatrics, Isfahan, Iran \\ 2Tehran University of Medical Sciences, Chronic Diseases Research Center, Endocrinology and Metabolism Population Sciences Institute, Tehran, Iran \\ ${ }^{3}$ Alborz University of Medical Sciences, Non-communicable Diseases Research Center, Karaj, Iran; Tehran University of Medical Sciences, \\ Endocrinology and Metabolism Research Center, Endocrinology and Metabolism Clinical Sciences Institute, Tehran, Iran \\ ${ }^{4}$ Ahvaz Jundishapur University of Medical Sciences, Department of Pediatrics, Ahvaz, Iran \\ ${ }^{5}$ Development of Research Technology Center, Deputy of Research and Technology, Ministry of Health and Medical Education, Tehran, Iran. \\ 6 Isfahan University of Medical Sciences, Medical Student, Isfahan, Iran \\ ${ }^{7}$ Tehran University of Medical Sciences, School of Nutritional Sciences and Dietetics, Department of Community Nutrition, Tehran, Iran
}

\begin{abstract}
Aim: Some evidence exists concerning the relationship between anthropometric measurements and chronic kidney disease. This study aims to investigate the association of neck circumferences (NC), wrist circumferences (WC) and hip (HC) circumferences with kidney function in a pediatric population.
\end{abstract}

Materials and Methods: In this national study, 4.200 students aged 7-18 years were selected by random cluster sampling from 30 provinces of Iran. NC, WC and HC were measured according to standard protocol and were categorized to either low or high according to their age-sex specific median values. The estimated glomerular filtration rate (eGFR) was calculated based on the "updated" Schwartz equation.

Results: The response rate was $91.5 \%$ ( $\mathrm{n}=3.843)$. The mean standard deviation of eGFR was 96.71 (19.46), 96.49 (21.69), and 96.59 (20.66) $\mathrm{mL} /$ minimum $/ 1.73 \mathrm{~m}^{2}$ for girls, boys and the total population, respectively. Compared to other participants, those in the high NC group had significantly higher eGFR $(102.12 \pm 21.31$ vs $90.65 \pm 18.18, p<0.001)$ and high creatinine $(\mathrm{Cr})(0.66 \pm 0.14 \mathrm{vs} 0.63 \pm 0.11 \mathrm{mg} / \mathrm{dL}, \mathrm{p}<0.001)$. Individuals categorized as high WC had significantly higher eGFR $(102.12 \pm 21.31$ vs $90.83 \pm 18.16, p<0.001)$ and $\operatorname{Cr}(0.66 \pm 0.15$ vs $0.63 \pm 0.10) \mathrm{mg} / \mathrm{dL}$, $\mathrm{p}<0.001)$. In the multivariate model, high NC, WC and HC were associated with higher eGFR ( $p<0.001)$. Moreover, each one-unit $(\mathrm{cm})$ increment in NC, WC and $\mathrm{HC}$ increased eGFR by $1.42,3.24$ and 0.46 units, respectively.

Conclusion: The findings of this large population-based study suggest that simple anthropometric measurements, such as WC and NCs, can be used in epidemiological studies to determine those children and adolescents that might be at risk of kidney dysfunction.

Keywords: Kidney function, neck circumference, wrist circumference, children, prevention

Mostafa Qorbani PhD, Alborz University of Medical Sciences, Non- Communicable Diseases Research Center, Karaj, Iran, \& Roya Kelishadi PdD, Isfahan University of Medical Sciences, Child Growth and Development Research Center, Research Institute For Primordial Prevention Of Non-communicable Disease, Department of Pediatrics, Isfahan, Iran Phone: +982188913543 E-mail: maorbani1379@yahoo.com \& roya.kelishadi@gmail.com ORCID: orcid.org/0000-0001-9465-7588 Received: 08.07.2018 Accepted: 11.02.2019 


\section{Introduction}

Chronic kidney disease (CKD) is an important health problem with complicated associations with other disorders such as cardiovascular diseases (1). Studies conducted in the United States (between 1988-1994 and 1999-2004) have reported that the prevalence of CKD is increasing from $10 \%$ to $13.1 \%$. Limited studies exist about CKD in non-Western populations. In Iran, its incidence is reported as $27.8 \%$ in females and $14.2 \%$ in males $(2,3)$.

CKD will cause a poor quality of life and also a huge economic burden as the patient may need kidney transplantation or dialysis (4). The disease is asymptomatic at the beginning and is not usually detected until the progression and development of complications. This makes the prevention of kidney failure or other outcomes very hard (5). Early detection of the disease can delay the progress to end stage renal disease or other severe complications (6).

Obesity is a growing worldwide problem in both developing and developed countries. It is a major determinant of most chronic disorders including $\operatorname{CKD}(7,8)$. Body mass index (BMI) is the most commonly used index for determining weight status. BMI is easy to calculate, but it has some limitations in describing body compositions. Waist circumference is largely used for determining the visceral obesity that is related to complications of obesity $(9,10)$. Measurement of waist circumference differs according to body position and breathing phase, and it is hard to measure it in routine primary care visits and some situations where adequate body exposure are difficult, therefore some other parameters including neck and wrist circumferences (WCs) are described and used in epidemiological studies (11). In the current study, we aim to determine the association of kidney function with neck and WCs in children and adolescents.

\section{Materials and Methods}

This multicentric cross-sectional study was conducted as part of the fifth survey of a national school-based surveillance program entitled "Childhood and Adolescence Surveillance and Prevention of Adult non-communicable Disease" study (2015). Detailed methodology has been described previously (12).

\section{Ethical Considerations}

The Research and Ethics Committee of Isfahan University of Medical Sciences approved this study (approval number: 194049). Participation in this study was voluntary. After a complete explanation of the study objectives and protocols, written informed consent and verbal consent were obtained from the parents and students, respectively.

\section{Study Population and Sampling Framework}

Participants consisted of students, aged 7-18 years, living in urban and rural areas of 30 provinces of Iran. They were selected by a multistage, stratified cluster sampling method. Using the proportional to size method and with an equal sex ratio, sampling within each of the provinces was conducted according to the student's area of residence (urban or rural) and level of education (primary and secondary). Moreover, the number of samples of different educational grades in urban and rural areas was estimated according to the number of students in each grade. The total sample size was calculated as 480 students in each province (48 clusters of 10 students); in each province, 14 clusters were randomly selected for biochemical testing, i.e. a total of 4.200 students.

\section{Procedure and Measurements}

Two sets of questionnaires were developed for students and their parents. The students' questionnaire was derived from the World Health Organization-Global School Student Health Survey. The validity and reliability of the Farsitranslated questionnaire was assessed previously $(13,14)$. During the interviews, not only demographic information, but also complementary information on physical activity (PA), screen time (ST), and socio-economic status, was completed for all participants.

Through the executive process of the survey, all examinations were conducted with calibrated instruments and the recording of information was completed through validated checklists which were designed and conducted under the standard protocol by trained health care professional teams $(15,16)$.

Neck circumference (NC), hip and WC were measured using a non-elastic tape to the nearest $0.1 \mathrm{~cm}$ over the skin. NC was measured by a tape underneath the Adam's apple in contact with the patient's skin in a comfortable position $(15,17)$.

WC was measured with subjects in a seated position for both wrists at distal to the prominences of the radial and ulnar and an average was taken $(18,19)$. Neck, wrist and hip were categorized as either low or high according to an agesex specific median.

\section{Blood Sampling}

Eligible students were referred to the laboratory, while one of the parents accompanied him/her. There, $6 \mathrm{~mL}$ 
venous blood samples were collected after 12-hr overnight fasting. All collection tubes were centrifuged at 2.5003.000 x g for 10 minutes. Immediately after centrifugation, serum samples were aliquot into 200 microliter tubes and stored at $-70{ }^{\circ} \mathrm{C}$. All samples were transferred by cold chain to the Isfahan Mahdieh Laboratory. Serum creatinine $(\mathrm{Cr})$ was measured enzymatically by the Hitachi auto-analyzer (Tokyo, Japan) $(20,21)$.

\section{Definition of Terms \\ Socio-economic Status}

The method, validity and considered variables for calculating the socio-economic status (SES) of Iranian families was approved previously through the Progress in the International Reading Literacy Study (22). Considering that, the principal component analysis of variables including parental education, parents' job, ownership of a private car, school type (public/private), and having a personal computer in the home were summarized in one main component. This component explained $72.0 \%$ of variance. This main component was categorized into tertials. The first tertial was defined as a low SES, the second tertial as an intermediate and the third tertial as a high SES.

\section{Screen Time}

To assess ST behaviors, the average number of hours per day that participants spent watching TV/VCDs, using personal computers (23), or playing electronic games was asked, then the total cumulative time spent for ST was estimated. Information was recorded separately for weekdays and weekends. The analysis of the correlates of ST was carried out according to the international ST recommendations and ST was categorized into two groups; less than 2 hours per day (low), and 2 hours per day or more (high) (24-26).

\section{Physical Activity}

Through a validated questionnaire, information regarding the past week's frequency of leisure time PA outside school was collected (12). PA was considered as at least a 30-minute duration of exercises that led to heavy sweating or a large increase in breathing or heart rate. Based on this, participants described their weekly PA habits via four available responses as follows; none, 1-2 days, 3-6 days, and every day. With the aim of analysis, weekly frequency of PA was categorized into three groups; less than two times per week (mild), two to four times a week (moderate) and more than 4 times a week (vigorous) (27).

\section{Glomerular Filtration Rate}

GFR describes the flow rate of filtered fluid through the kidney (28). The estimated eGFR is used to screen for the early detection of kidney damage, to help diagnose CKD, and to monitor kidney status. It is a calculation based on the results of a blood $\mathrm{Cr}$ test adjusted for age and sex based on the equation used (28). In the present study, eGFR was calculated based on the "updated" Schwartz equation formula (29):

$$
\mathrm{eGFR}=\frac{0.413 \times \text { height }(\mathrm{cm})}{\text { serum creatinine }\left(\frac{\mathrm{mg}}{\mathrm{dL}}\right)}
$$

\section{Statistical Analysis}

Continuous and categorical variables are expressed as mean [standard deviation (SD)] and number (percentage) respectively. The Kolmogorov-Smirnov test was used to examine the normality of continuous variables. Associations of continuous and categorical variables with age groups were compared by ANOVA and the chi-square test, respectively.

The mean of eGFR and $\mathrm{Cr}$ across categorized levels of hip, neck, and WCs was compared by t-test. Linear regression analysis was used to examine the association of hip, neck and WCs with eGFR and Cr.

Three models were applied: Model I: the crude model (without adjustment); Model II: was adjusted for age, area of residence (urban or rural), sex, PA, ST and SES; and Model III: was additionally adjusted for BMI. All statistical analyses were performed using a survey analysis method, and were conducted using the statistical program STATA package version 11.0 (stata statistical software: Release 11. College Station, TX: StataCorp LP. Package). P values of less than 0.05 were considered as statistically significant.

\section{Results}

The study participants consisted of 3.843 students with a mean age of $12.28 \pm 3.15$ years, without any significant difference between boys and girls. From them, 50.6\% were boys and $71.4 \%$ were from urban areas. The characteristics of the participants are presented in Table I. It shows that PA and SES were significantly different between the age groups. Whereas, eGFR and $\mathrm{Cr}$, respectively, with an overall mean \pm SD of $96.59 \pm 20.66$ and $0.65 \pm 0.14(\mathrm{mg} / \mathrm{dL})$, showed significant ascending differences between the age groups ( $p$-trend<0.001). Likewise, BMI, NC, WC and HC, with means of $18.51 \pm 4.71\left(\mathrm{~kg} / \mathrm{m}^{2}\right), 29.84 \pm 3.99(\mathrm{~cm}), 14.72 \pm 1.89(\mathrm{~cm})$, and $79.14 \pm 14.64(\mathrm{~cm})$ followed an ascending trend with increasing age ( $p$-trend $<0.001)$. 
Considering the mean \pm SD of eGFR and $\mathrm{Cr}$ according to NC, WC and HC; participants in the high NC group had significantly higher eGFR (102.12 \pm 21.31 vs $90.65 \pm 18.18$, $\mathrm{p}<0.001$ ) and $\mathrm{Cr}$ (high; $0.66 \pm 0.14$ vs $0.63 \pm 0.11 \mathrm{mg} / \mathrm{dL}$, $\mathrm{p}<0.001)$. Except for $\mathrm{Cr}$ levels in girls, these significant associations were also documented in other groups. Likewise, those participants who were categorized as the high WC group had significantly higher eGFR $(102.12 \pm 21.31$ vs $90.83 \pm 18.16, p<0.001)$ and $\mathrm{Cr}[(0.66 \pm 0.15$ vs $0.63 \pm 0.10) \mathrm{mg} /$ $\mathrm{dL}, \mathrm{p}<0.001]$ than their counterparts. Except for $\mathrm{Cr}$ levels of girls, this significant association existed in other groups as well. Regarding the two groups of high and low HC, both boys and girls had higher eGFR in the high HC groups (boys: 103.66 \pm 22.38 , girls: $102.10 \pm 19.43 \mathrm{mg} / \mathrm{dL}, \mathrm{p}<0.001$ ) (Table II).
Table III shows the association of NC, WC and HC as continuous and categorical variables with eGFR and $\mathrm{Cr}$ in linear regression analysis. In a multivariate model, NC, WC and HC, as continuous and categorical variables, were associated with eGFR; participants with high NC, WC and HC, compared with their other counterparts, had significantly higher eGFR ( $p<0.001)$. In a multivariate model (Model III), each one unit $(\mathrm{cm})$ increment in NC, WC and HC increased eGFR by 1.42, 3.24 and 0.46 units, respectively.

The multivariate model on the association of NC, WC and $\mathrm{HC}$, as continuous and categorical variables, with $\mathrm{Cr}$, showed that only continuous NC and WC were associated with $\mathrm{Cr}$ levels; per each one unit $(\mathrm{cm})$ increment in $\mathrm{NC}$ and $\mathrm{WC}, \mathrm{Cr}$ increased significantly by 0.002 and $0.004 \mathrm{mg} / \mathrm{dL}$, respectively.

Table I. Characteristics of participants by gender and age group: The CASPIAN-V study

\begin{tabular}{|c|c|c|c|c|c|}
\hline & \multicolumn{3}{|c|}{ Age } & \multirow{2}{*}{ Total } & \multirow{2}{*}{$p$ value } \\
\hline & $7-10 y$ & $11-14 y$ & $15-18 y$ & & \\
\hline Mean age (year) ${ }^{1}$ & $8.74 \pm 1.03$ & $12.51 \pm 1.08$ & $16.41 \pm 1.04$ & $12.28 \pm 3.15$ & $<0.001$ \\
\hline \multicolumn{6}{|l|}{ Sex ${ }^{2}$} \\
\hline Boy & $2.358(48.7)$ & $2.826(50.5)$ & $2.044(53.2)$ & $7.228(50.6)$ & \multirow{2}{*}{$<0.001$} \\
\hline Girl & $2.485(51.3)$ & $2.765(49.5)$ & $1.796(46.8)$ & $7.046(49.4)$ & \\
\hline \multicolumn{6}{|l|}{ Area of residence } \\
\hline Urban & $3.235(66.8)$ & $3.683(65.9)$ & $3,276(85.3)$ & $10.194(71.4)$ & \multirow{2}{*}{$<0.001$} \\
\hline Rural & $1.608(33.2)$ & $1.908(34.1)$ & $564(14.7)$ & $4.080(28.6)$ & \\
\hline BMI $\left(\mathrm{kg} / \mathrm{m}^{2}\right)^{1}$ & $16.18 \pm 4.03$ & $18.99 \pm 4.41$ & $21.21 \pm 4.42$ & $18.51 \pm 4.71$ & $<0.001$ \\
\hline \multicolumn{6}{|l|}{ Physical activity ${ }^{2}$} \\
\hline Low & $1.470(32.6)$ & $1.683(32.3)$ & $1.301(36.1)$ & $4.454(33.4)$ & \multirow{3}{*}{0.001} \\
\hline Medium & $1.482(32.9)$ & $1.784(34.3)$ & $1.158(32.1)$ & $4.424(33.2)$ & \\
\hline High & $1.554(34.5)$ & 1.739 (33.4) & $1.147(31.8)$ & $4.440(33.3)$ & \\
\hline \multicolumn{6}{|l|}{ Screen Time ${ }^{2}$} \\
\hline Low & $4.023(85.4)$ & $4.553(84.2)$ & $3.068(81.5)$ & $11.644(83.8)$ & \multirow{2}{*}{$<0.001$} \\
\hline High & $688(14.6)$ & $857(15.8)$ & $698(18.5)$ & $2.243(16.2)$ & \\
\hline \multicolumn{6}{|l|}{ SES $^{2}$} \\
\hline Low & 1.524 (32.9) & $1.830(34.4)$ & $1.205(32.8)$ & $4.454(33.4)$ & \multirow{3}{*}{0.06} \\
\hline Medium & $1.514(32.7)$ & $1.723(32.4)$ & $1.278(34.8)$ & $4.424(33.2)$ & \\
\hline High & $1.595(34.4)$ & $1.764(33.2)$ & $1.193(32.5)$ & $4.440(33.3)$ & \\
\hline Neck circumference $^{1}$ & $27.25 \pm 3.06$ & $29.97 \pm 3.42$ & $32.93 \pm 3.54$ & $29.84 \pm 3.99$ & $<0.001$ \\
\hline Waist circumference ${ }^{1}$ & $13.44 \pm 1.47$ & $14.92 \pm 1.62$ & $16.03 \pm 1.71$ & $14.72 \pm 1.89$ & $<0.001$ \\
\hline Hip circumference ${ }^{1}$ & $69.08 \pm 10.57$ & $80.32 \pm 1245$ & $90.18 \pm 13.39$ & $79.14 \pm 14.64$ & $<0.001$ \\
\hline eGFR1 & $87.23 \pm 15.72$ & $99.30 \pm 19.76$ & $102.60 \pm 23.20$ & $96.59 \pm 20.66$ & $<0.001$ \\
\hline Creatinine $(\mathrm{mg} / \mathrm{dL})^{1}$ & $0.63 \pm 0.10$ & $0.64 \pm 0.11$ & $0.69 \pm 0.16$ & $0.65 \pm 0.14$ & $<0.001$ \\
\hline
\end{tabular}

${ }^{1}$ Data are presented as mean (standard deviation), ${ }^{2}$ Data are presented as number (percentage)

CASPIAN-V: BMI: Body mass index, SES: Socio-economic status, eGFR: Eestimated glomerular filtration rate, $0.413^{*}$ height $(\mathrm{cm}) /$ serum creatinine $(\mathrm{mg} / \mathrm{dL})$ 
Table II. Mean \pm standard deviation of estimated glomerular filtration rate and creatinine according hip, neck and wrist circumference: The CASPIAN-V study

\begin{tabular}{|c|c|c|c|c|c|c|}
\hline \multirow[t]{2}{*}{ Variable } & \multicolumn{3}{|c|}{ eGFR } & \multicolumn{3}{|c|}{ Creatinine (mg/dL) } \\
\hline & Total & Boy & Girl & Total & Boy & Girl \\
\hline \multicolumn{7}{|c|}{ Neck circumference ${ }^{a}$} \\
\hline Low & $90.65 \pm 18.18$ & $90.02 \pm 19.07$ & $91.31 \pm 17.17$ & $0.63 \pm 0.10$ & $0.64 \pm 0.11$ & $0.62 \pm 0.10$ \\
\hline High & $102.12 \pm 21.31$ & $102.28 \pm 22.34$ & $101.99 \pm 20.08$ & $0.66 \pm 0.14$ & $0.67 \pm 0.14$ & $0.66 \pm 0.15$ \\
\hline$p$ value & $<0.001$ & $<0.001$ & $<0.001$ & $<0.001$ & $<0.001$ & 0.008 \\
\hline \multicolumn{7}{|c|}{ Wrist circumference ${ }^{b}$} \\
\hline Low & $90.83 \pm 18.16$ & $89.76 \pm 19.24$ & $91.87 \pm 16.97$ & $0.63 \pm 0.10$ & $0.64 \pm 0.11$ & $0.62 \pm 0.10$ \\
\hline High & $102.12 \pm 21.33$ & $102.18 \pm 22.07$ & $102.04 \pm 20.39$ & $0.66 \pm 0.15$ & $0.66 \pm 0.16$ & $0.66 \pm 0.14$ \\
\hline$p$ value & $<0.001$ & $<0.001$ & $<0.001$ & $<0.001$ & $<0.001$ & 0.007 \\
\hline \multicolumn{7}{|c|}{ Hip circumference ${ }^{c}$} \\
\hline Low & $89.78 \pm 17.98$ & $89.17 \pm 18.3$ & $90.49 \pm 17.53$ & $0.63 \pm 0.11$ & $0.64 \pm 0.11$ & $0.62 \pm 0.10$ \\
\hline High & $102.89 \pm 20.99$ & $103.66 \pm 22.38$ & $102.10 \pm 19.43$ & $0.66 \pm 0.15$ & $0.66 \pm 0.14$ & $0.66 \pm 0.15$ \\
\hline$p$ value & $<0.001$ & $<0.001$ & $<0.001$ & $<0.001$ & 0.004 & 0.005 \\
\hline
\end{tabular}

aAccording to age-sex specific median, ${ }^{b}$ According to age-sex specific median, 'According to age-sex specific median, CASPIAN-V: eGFR: estimated glomerular filtration rate

\section{Discussion}

As the first study of its kind in a non-Western population, we investigated the association between some anthropometric measurements including hip, neck and WCs with renal function in a large national pediatric population. The results demonstrated that in different age groups of girls and boys, those participants with lower hip, neck and WCs had better kidney function than their counterparts. Adjusted models of logistic regression analysis showed that the association between GFR and the afore mentioned anthropometric measurements was more prominent than that of $\mathrm{Cr}$.

Recently, the evaluation of the associations between anthropometric indices, including neck and WCs, and disease-related biological markers have gained more interest; which is due to its low cost and non-invasive method of measurement (30).

$\mathrm{NC}$ is considered as the representative anthropometric parameter of upper-body subcutaneous fat (31). The appropriate inter and intra reliability of NC among 6-16 year-old children and adolescents have been reported on previously (32). Accordingly, multiple measurements are not necessary for this index. In addition, its measurement has a simple method that can be easily performed by health care professionals (32).

Some studies have indicated the association between NC and obesity, cardio metabolic risk factors and insulin resistance in children $(33,34)$. Moreover, some studies demonstrated that the association between NC and cardiometabolic risk factors is more significant than other anthropometric parameters such as waist circumference or BMI (35).

Although some studies exist regarding the usefulness of measuring $\mathrm{NC}$ for predicting renal function in adults, to the best of our knowledge, there is no study in this field based on a pediatric population.

Recently, Yoon et al. (36) in a prospective cohort study (Korean Genome and Epidemiology Study cohort) have evaluated the association between NC and incident CKD. They revealed that $N C$ could be used as an independent predictor for CKD. Their observed association persisted even after adjustment for other anthropometric measurements such as BMI and waist-to-hip ratio, baseline eGFR and traditional risk factors of CKD. They also found that this association would be more prominent in the presence of obesity and elevated BMI.

The findings of the Liu et al. (37) study suggested that NC, as an indicator of upper-body subcutaneous fat, could have a pathogenic role in the occurrence of renal dysfunction. They showed that $\mathrm{NC}$ is associated with other indicators of renal function including uric acid, micro albuminuria, 24-hr $\mathrm{Cr}$ clearance rate and eGFR based on the Cockcroft and Gault formula as well as cardiovascular risk factors such as serum lipid levels and hs-C-reactive protein. 
Table III. Association of hip, neck and wrist circumference with estimated Glomerular Filtration Rate and Creatinine in logistic regression analysis: The CASPIAN-V study

\begin{tabular}{|c|c|c|c|c|c|c|}
\hline & \multicolumn{3}{|c|}{ eGFR } & \multicolumn{3}{|c|}{ Creatinine (mg/dL) } \\
\hline & $\beta$ & SE & $\mathrm{p}$ value & $\beta$ & SE & $p$ value \\
\hline \multicolumn{7}{|c|}{ Neck circumference $(\mathrm{cm})$} \\
\hline Model I & 1.42 & 0.08 & $<0.001$ & 0.004 & 0.001 & $<0.001$ \\
\hline Model II & 0.88 & 0.10 & $<0.001$ & 0.001 & 0.001 & 0.03 \\
\hline Model III & 0.80 & 0.11 & $<0.001$ & 0.002 & 0.001 & 0.01 \\
\hline \multicolumn{7}{|c|}{ Neck circumference, High/Lowa } \\
\hline Model I & 11.47 & 0.64 & $<0.001$ & 0.03 & 0.007 & $<0.001$ \\
\hline Model II & 6.73 & 0.80 & $<0.001$ & 0.004 & 0.005 & 0.45 \\
\hline Model III & 6.55 & 0.86 & $<0.001$ & 0.004 & 0.005 & 0.43 \\
\hline \multicolumn{7}{|c|}{ Wrist circumference $(\mathrm{cm})$} \\
\hline Model I & 3.24 & 0.17 & $<0.001$ & 0.01 & 0.002 & $<0.001$ \\
\hline Model II & 2.07 & 0.22 & $<0.001$ & 0.003 & 0.001 & 0.01 \\
\hline Model II & 2.17 & 0.25 & $<0.001$ & 0.004 & 0.001 & 0.006 \\
\hline \multicolumn{7}{|c|}{ Wrist circumference, High/Lowb } \\
\hline Model I & 11.29 & 0.64 & $<0.001$ & 0.03 & 0.007 & $<0.001$ \\
\hline Model II & 7.15 & 0.78 & $<0.001$ & 0.002 & 0.005 & 0.63 \\
\hline Model III & 7.07 & 0.84 & $<0.001$ & 0.003 & 0.005 & 0.62 \\
\hline \multicolumn{7}{|c|}{ Hip circumference $(\mathrm{cm})$} \\
\hline Model I & 0.46 & 0.02 & $<0.001$ & 0.001 & 0.00 & $<0.001$ \\
\hline Model II & 0.34 & 0.02 & $<0.001$ & 0.00 & 0.00 & 0.62 \\
\hline Model II & 0.38 & 0.03 & $<0.001$ & 0.00 & 0.00 & 0.51 \\
\hline \multicolumn{7}{|c|}{ Hip circumference, High/Lowc } \\
\hline Model I & 13.11 & 0.63 & $<0.001$ & 0.03 & 0.007 & $<0.001$ \\
\hline Model II & 8.93 & 0.81 & $<0.001$ & -0.002 & 0.005 & 0.64 \\
\hline Model III & 9.18 & 0.22 & $<0.001$ & -0.002 & 0.006 & 0.67 \\
\hline
\end{tabular}

aAccording to age-sex specific median, ${ }^{b}$ According to age-sex specific median, ${ }^{c}$ According to age-sex specific median, Model I: Without adjustment, Model II: Adjusted for age, living area, sex, physical activity and screen time, SES, Model III: Additionally adjusted for BMI eGFR: Estimated glomerular filtration rate, SES: Socio-economic status

To the best of our knowledge, the current findings are the first to show a significant association between NC in children and GFR, which is considered as the most important and earliest indicator of renal dysfunction. It seems that this anthropometric index could be applicable both in epidemiological studies and clinical practice for screening for renal dysfunction in the pediatric population.

The association between obesity and the development of CKD has been documented in some studies $(38,39)$. Moreover, the relationship between obesity and end stage renal disease has a direct correlation irrespective of underlying factors such as hypertension or diabetes (39).
WC is another simple anthropometric measurement, which has a safe and non-invasive method with appropriate intra- and inter-operator reliability (40). In addition, it is related to skeletal frame size, and is not affected by body fat variations (40).

A study in Iranian adults reported that WC, as a novel anthropometric measurement, can be considered as an independent predictor for incident hypertension and cardiovascular disease among non-centrally obese women (41). However, in another study in the same population, WC had significant correlation with lipid profile, but not with metabolic syndrome or cardiovascular diseases (42). 
Some other studies indicated that WC is associated with insulin resistance both in children and adults (43), as well as with diabetes in the adult population (44).

A recent study in Italy showed that WC could be used as an indicator of insulin resistance in obese youth (40). A cohort with 30 years of follow up showed that this measurement is an indicator of insulin resistance and BMI in children but not in adults (45).

Thus, considering the association between obesity, insulin resistance and abnormal glucose metabolism with $C K D$, as well as the current finding on the association between WC and GFR, it is suggested that WC can be used as a predictor of CKD in children.

In this study, although the mean serum $\mathrm{Cr}$ level was higher in those participants with higher measurements of hip, neck and WCs, it had no significant correlation with these anthropometric measurements. In addition, these very small differences are of no clinical importance.

This investigation had a cross-sectional design, which is considered as its main limitation. In addition, the results of this study would be more applicable if the association of the afore mentioned anthropometric indices with other markers of kidney function, such as cystatin C, had been investigated.

This study was conducted as a part of a national study with a large sample size and, to the best of our knowledge, this was the first national study in the pediatric population which has evaluated the association of neck and WCs with renal function.

\section{Conclusion}

The findings of this large population-based study suggest that both neck and WCs are appropriate, simple, non-invasive and easy to detect anthropometric measurements that can be used in epidemiological and clinical studies for determining those children and adolescents who are at risk of kidney dysfunction.

\section{Ethics}

Ethics Committee Approval: The Research and Ethics Committee of Isfahan University of Medical Sciences approved this study (approval number: 194049).

Informed Consent: Written informed consent and verbal consent were obtained from the parents and students, respectively.

Peer-review: Externally peer-reviewed.

\section{Authorship Contributions}

Surgical and Medical Practices: R.K., M.E.M., Concept: R.H., M.E.M., M.Q., R.K., Design: R.H., M.Q., R.K., Data Collection or Processing: M.T., T.A., G.S., A.M.G., S.Z., Analysis or Interpretation: M.Q., Literature Search: A.M.G., S.D., A.A., Writing: M.M., A.M.G.

Conflict of Interest: The authors have no conflicts of interest relevant to this article to disclose.

Financial Disclosure: The authors have no financial relationships relevant to this article to disclose.

\section{References}

1. Snively CS, Gutierrez C. Chronic kidney disease: Prevention and treatment of common complications. Ame Fam Physician 2004;70:1921-8

2. Coresh J, Selvin E, Stevens LA, et al. Prevalence of chronic kidney disease in the United States. JAMA 2007;298:2038-47.

3. Tohidi M, Hasheminia M, Mohebi $R$, et al. Incidence of chronic kidney disease and its risk factors, results of over 10 year follow up in an Iranian cohort. Plos one 2012;7:45304.

4. Smith DH, Gullion CM, Nichols G, Keith DS, Brown JB. Cost of medical care for chronic kidney disease and comorbidity among enrollees in a large HMO population. I Am Soc Nephrol I Am Soc Nephrol 2004;15:1300-6.

5. Locatelli F, Vecchio LD, Pozzoni P. The importance of early detection of chronic kidney disease. Nephrol Dial Transplant 2002;11(Suppl):2-7.

6. He Y, Li F, Wang F, Ma X, Zhao X, Zeng Q. The association of chronic kidney disease and waist circumference and waistto-height ratio in Chinese urban adults. Medicine (Baltimore) 2016;95:e3769.

7. Byers T. Body weight and mortality. N Engl J Med 1995;333:723-4.

8. Kovesdy CP, Czira ME, Rudas A, et al. Body mass index, waist circumference and mortality in kidney transplant recipients. Am J Transplant 2010;10:2644-51.

9. Misra A, Chowbey P, Makkar BM, et al. Consensus statement for diagnosis of obesity, abdominal obesity and the metabolic syndrome for Asian Indians and recommendations for physical activity, medical and surgical management. ) Assoc Physicians India 2009:57:163-70

10. Chan DC, Watts GF, Barrett PH, Burke V. Waist circumference, waist-to-hip ratio and body mass index as predictors of adipose tissue compartments in men. QJM 2003;96:441-7.

11. Karki BB, Bhattarai MD, Bajracharya MR, Karki S, Devkota AR. Correlation of neck and wrist circumference with waist circumference. IAIM 2015;3:47-51.

12. Kelishadi R, Majdzadeh R, Motlagh ME, et al. Development and evaluation of a questionnaire for assessment of determinants of weight disorders among children and adolescents: The Caspian-IV study. Int J Prev Med 2012;3:699-705.

13. Kelishadi R, Majdzadeh R, Motlagh ME. Development and evaluation of a questionnaire for assessment of determinants of weight disorders among children and adolescents: The Caspian-IV study. Int I Prev Med 2012;3:699-705. 
14. Organization WH. Expert committee on physical status. Physical Status: The use and interpretation of anthropometry Geneva: WHO, 1995.

15. WHO Multicentre Growth Reference Study Group. WHO Child Growth Standards based on length/height, weight and age. Acta Paediatr Suppl 2006;450:76-85

16. Motlagh ME, Ziaodini $H$, Qorbani M, et al. Methodology and early findings of the fifth survey of childhood and adolescence surveillance and prevention of adult noncommunicable disease: The caspian-v study. Int J Prev Med 2017;8:4.

17. Knowles KM, Paiva LL, Sanchez SE, et al. Waist circumference, body mass index, and other measures of adiposity in predicting cardiovascular disease risk factors among peruvian adults. Int I Hypertens 2011;2011:931402.

18. Kuczmarski RJ, Ogden CL, Grummer-Strawn LM, Flegal KM, et al. CDC growth charts: United States. Adv Data 2000:1-27.

19. Kelishadi RSG, Heshmat R, Djalalinia S, et al. Wrist circumference as a novel predictor of obesity in children and adolescents: The CASPIAN IV study. I Am Soc Hypertens 2014;8:8.

20. McNamara IR, Schaefer E). Automated enzymatic standardized lipid analyses for plasma and lipoprotein fractions. Clin Chim Acta 1987;166:1-8.

21. Friedewald WT, Levy RI, Fredrickson DS. Estimation of the concentration of low-density lipoprotein cholesterol in plasma, without use of the preparative ultracentrifuge. Clin Chem 1972;18:499-502.

22. Caro DH, Cortés D. Measuring family socioeconomic status: An illustration using data from PIRLS 2006. lerinstitue 2012;5:9-33.

23. Stefanowicz M, Strączkowski M, Karczewska-Kupczewska M. The role of SIRT1 in the pathogenesis of insulin resistance in skeletal muscle. Postepy Hig Med Dosw (Online) 2015;69:63-8.

24. Salmon I, Campbell K, Crawford DA. Television viewing habits associated with obesity risk factors: A survey of Melbourne schoolchildren. Med ) Aust 2006;184:64-7.

25. American Academy of Pediatrics. Committee on Public Education. American Academy of Pediatrics: Children, adolescents, and television. Pediatrics 2001;107:423-6.

26. Emamian $\mathrm{MH}$, Zeraati $\mathrm{H}$, Majdzadeh $\mathrm{R}$, et al. Economic inequality in presenting near vision acuity in a middle-aged population: $\mathrm{A}$ Blinder-oaxaca decomposition. Br J Ophthalmol 2013;97:1100-3.

27. Drenowatz C, Carlson JJ, Pfeiffer KA, Eisenmann JC. Joint association of physical activity/screen time and diet on CVD risk factors in 10-year-old children. Front Med 2012;6:428-35.

28. Stevens LA, Coresh I, Greene T, Levey AS. Assessing kidney function-measured and estimated glomerular filtration rate. $\mathrm{N}$ Engl ) Med 2006;354:2473-83.

29. Schwartz G), Muñoz A, Schneider MF, et al. New equations to estimate GFR in children with CKD. I Am Soc Nephrol I Am Soc Nephrol 2009;20:629-37.

30. Petkeviciene J, Klumbiene J, Kriaucioniene V, Raskiliene A, Sakyte E, Ceponiene I. Anthropometric measurements in childhood and prediction of cardiovascular risk factors in adulthood: Kaunas cardiovascular risk cohort study. BMC Public Health 2015;15:218
31. Preis SR, Massaro JM, Hoffmann $U$, et al. Neck circumference as a novel measure of cardiometabolic risk: The Framingham Heart study. I Clin Endocrinol Metab 2010;95:3701-10.

32. LaBerge RC, Vaccani JP, Gow RM, Gaboury I, Hoey L, Katz $\mathrm{SL}$. Inter-and intra-rater reliability of neck circumference measurements in children. Pediatr Pulmonol 2009;44:64-9.

33. da Silva Cde C, Zambon MP, Vasques AC, et al. Neck circumference as a new anthropometric indicator for prediction of insulin resistance and components of metabolic syndrome in adolescents: Brazilian Metabolic Syndrome Study. Rev Paul Pediatr 2014;32:221-9.

34. Kim Y, Lee JM, Laurson K, Bai Y, Gaesser GA, Welk G). Accuracy of neck circumference in classifying overweight and obese US children. ISRN Obes 2014;2014:781841.

35. Zhou JY, Ge $H$, Zhu MF, et al. Neck circumference as an independent predictive contributor to cardio-metabolic syndrome. Cardiovascular Diabetol 2013;12:76.

36. Yoon CY, Park JT, Jhee JH, et al. Neck circumference predicts renal function decline in overweight women: A community-based prospective cohort study. Medicine (Baltimore) 2016;95:e4844.

37. Liu YF, Chang ST, Lin WS, et al. Neck circumference as a predictive indicator of CKD for high cardiovascular risk patients. Biomed Res Int 2015;2015:745410.

38. Hsu CY, McCulloch CE, Iribarren C, Darbinian J, Go AS. Body mass index and risk for end-stage renal disease. Ann Intern Med 2006;144:21-8.

39. Vivante A, Golan E, Tzur D, et al. Body mass index in 1.2 million adolescents and risk for end-stage renal disease. Arch Intern Med 2012;172:1644-50.

40. Campagna G, Zampetti S, Gallozzi A, et al. Excellent intra and inter-observer reproducibility of wrist circumference measurements in obese children and adolescents. Plos One 2016;11:e0156646.

41. Mohebi R, Mohebi A, Sheikholeslami F, Azizi F, Hadaegh F. Wrist circumference as a novel predictor of hypertension and cardiovascular disease: Results of a decade follow up in a West Asian cohort. J Am Soc Hypertens 2014;8:800-7.

42. Hajsadeghi S, Firouzi A, Bahadoran P, Hassanzadeh M. The value of wrist circumference for predicting the presence of coronary artery disease and metabolic syndrome. Indian Heart I 2016;68(Suppl 3):5-9.

43. Mueller NT, Johnson W, Odegaard AO, Lee M, Czerwinski SA, Demerath EW. Wrist breadth and homeostasis model assessment of insulin resistance in youth: The Fels Longitudinal Study. Am J Hum Biol 2013;25:581-5.

44. Jahangiri Noudeh $Y$, Hadaegh F, Vet al. Wrist circumference as a novel predictor of diabetes and prediabetes: Results of cross-sectional and 8.8-year follow-up studies. J Clin Endocrinol Metab 2013;98:777-84

45. Watkins AN, Kelly AS, Prineas RI, et al. Childhood wrist circumference is not a predictor of insulin resistance in adulthood. J Pediatr 2015;166:1085-7. 